\title{
陇东黄土高原石鸡的遗传多样性与保护
}

\author{
黄族豪 ${ }^{1}$ 刘迺发 $^{1 *}$ 周天林 ${ }^{2}$ \\ 1 (兰州大学生命科学学院, 兰州 730000) \\ 2 ( 甘肃省陇东学院生物系, 庆阳 744000)
}

摘要 : 石鸡 (Alectoris chukar) 是我国北方重要的猎鸟, 由于栖息地片断化和人类狩猎, 陇东黄土高原上的石鸡数量 正日益减少。本文用 PCR 直接测序的方法, 测定了陇东黄土高原 8 个石鸡种群 mtDNA 控制区 I 区和部分 II 区的 491 个碱基, 探讨其遗传多样性。78 个样本共发现 24 个变异位点 (占所测序列的 4.89\%)和 25 种单倍型 (占所测 样本的 $32.05 \%) 。 8$ 个种群中, 铜川种群的序列变异率、单倍型多样性和核苷酸多样性都最高, 分别是 $0.47 、 0.82$ 和 0.0029 ; 而红回种群的最低, 仅分别为 $0.10 、 0.28$ 和 0.0008 , 这与红回种群受奠基者效应、遗传隔离和自然选择 的作用有关。 8 个种群共享 1 种单倍型 $\mathrm{C} 1$, 说明它们来自共同祖先, 是 1 个单系群, 属于 1 个进化显著单元, 但它们 聚成两个集群。两集群间单倍型相似性指数仅 0.15 , 遗传距离达 $0.43 \%$, 单因素方差分析显示遗传变异差异显著 $\left(F=5.02>\mathrm{F}_{0.05}(14,1)=4.06\right)$, 分别有 13 种和 10 种单倍型为两个集群所特有。基于遗传差异性, 陇东黄土高原 的石鸡应分为两个管理单元进行保护, 尤其对遗传变异和遗传多样性最高的铜川种群应进行重点保护。

关键词 : Alectoris chukar, 线粒体 DNA, 单倍型, 进化显著单元, 管理单元

中图分类号:Q16 文献标识码：A 文章编号 : 1005-0094(2003)06-0454-07

\section{Genetic diversity and protection of chukar partridge (Alectoris chukar) on the Loess Plateau of eastern Gansu}

\author{
HUANG Zu-Hao ${ }^{1}$, LIU Nai-Fa ${ }^{1 *}$, ZHOU Tian-Lin ${ }^{2}$ \\ 1 College of Life Sciences, Lanzhou University, Lanzhou 730000 \\ 2 Department of Biology, Longdong College, Qingyang , Gansu 744000
}

Abstract : Chukar partridge (Alectoris chukar) is a very important bird for hunting in northern China. Currently chukar partridges are threatened by habitat loss and fragmentation, hunting, and human persecution on the Loess Plateau of eastern Gansu. We used polymerase chain reaction and dideoxy direct sequencing methods to analyze the genetic diversity of chukar partridge. A total of 491 nucleotides of the mitochondrial DNA control region gene were sequenced in 78 chukar partridges of eight populations collected from the Loess Plateau. The 24 variable sites (4.89\% of the entire sequences) defined 25 haplotypes $(32.05 \%$ of all the samples). The sequence variation, haplotype diversity and nucleotide diversity of the Tongchuan population were the largest among the eight populations, up to $0.47,0.82$ and 0.0029 , respectively, while those of Honghui population were the lowest , $0.10,0.28$ and 0.0008 , respectively. This might result from founder effect, genetic isolation or natural selection. The eight populations shared one haplotype $\mathrm{C} 1$, which indicated that they came from a common ancestor and belonged to one monophyletic group and one evolutionarily significant unit. However, they clustered into two groups. The haplotype similarity index between the two groups was only 0.15 , and genetic distance was $0.43 \%$. The genetic variability was significantly different between the two groups according to one-way analysis of variance $\left(F=5.02>\mathrm{F}_{0.05}(14,1)=4.06\right)$. It is suggested that the chukar partridge throughout the Loess Plateau of eastern Gansu should be protected as two management units since the demographic connec- 
tion between them is low. The Tongchuan population, with the highest genetic variation and genetic diversity, should be well-protected.

Key words : Alectoris chukar, mitochondrial DNA, haplotype, evolutionarily significant units , management units

遗传多样性是指种内不同种群之间或同一种群 内的遗传变异（施立明等，1993），它是生物多样性 的核心内容 (Humphries et al. ，1995)。制定有效的 保护对策应以保护物种的遗传多样性为中心 (王静 波等, 2001)。在现代保护生物学中, 遗传学分析占 据非常重要的地位。保护生物学与分子生物学相结 合已成为当前的研究热点 (Haig ,1998)。分子标记 的应用有助于人们更清楚地了解种群间关系及其进 化历史，从而为物种保护提供更精确的科学依据 (杨玉慧，李义明，2001）。传统的种和亚种概念多 基于地理分布和形态，而没有考虑遗传多样性，不宜 作为生物保护的基本单元 ( O'Brien \& Mayr , 1991)。 而基本保护单元的确定是优化和实施保护策略的基 础 (王文, 1998)。Ryder (1986) 最早提出生物保护 中进化显著单元 ( evolutionarily significant units, ESUs) 的思想 , Moritz ( 1994 ) 则提出管理单元 (management units, MUs) 的概念。ESUs 和 MUs 对制定切实 可行的保护对策有重要的参考和应用价值 (Avise， 1994 ）,已被国际社会普遍接受为生物保护中的基 本单元 (王静波等,2001)。国内对 ESUs 和 MUs 的 实际研究较少, 至今未见鸟类这方面的研究报道。 线粒体 DNA (mtDNA) 进化速度快, 是单拷贝核 DNA (nDNA) 的 5 10 倍 (Brown et al. , 1979) , 并且严格 遵守母系遗传 (Gyllensten et al. ，1985)，所有的变 异都来自于突变, 常被用来确定 ESUs 和 MUs。

石鸡 (Alectoris chukar) 是我国北方的著名猎鸟， 具有重要的经济价值 (王香亭, 1991) 和科研价值 ( Randi \& Alkon ,1994)。陇东黄土高原上的石鸡华 北亚种 (A. c. pubescens) 是我国的特有亚种。由于栖 息地片断化和人类狩猎,其数量正日益减少。本文 利用 PCR 直接测序的方法测定了陇东高原 8 个种 群 78 个石鸡样本的 $\mathrm{mtDNA}$ 控制区的 $491 \mathrm{bp}$, 以探 讨其遗传多样性与保护策略。

\section{1 材料与方法}

\section{1 实验材料}

石鸡采自甘肃环县甜水 ( TS ) 、环县西川 ( XC) 、
环县曲子 $(Q Z)$ 、庆阳铜川 $(T C)$ 、宁县盘克 $(P K)$ 、泾 川高平 (GP)、华亭王峡 (WX) 和宁夏海原红回 ( $\mathrm{HH}$ ) (图 1) , 共 8 个种群 78 个个体。以石鸡的肝 脏和血液为实验材料,肝脏用 $95 \%$ 乙醇保存, 血液 用 $5 \%$ 肝素钠抗凝。

\subsection{DNA 提取, PCR 反应和 DNA 测序}

DNA 提取采用乙醇沉淀法, 参照 Randi \& Luc$\operatorname{chini}(1998)$ 。用引物 PHDL (5'-AGGACTACGGCTTGAAAAGC-3') 和 PH1H ( $5^{\prime}$-TTATGTGCTTGACCGAGGAACCAG-3') ( Randi \& Lucchini, 1998) 进行 $\mathrm{PCR}$ 反应, 体积为 $35 \mu \mathrm{L}$, 反应液中含 $10 \mathrm{mmol} / \mathrm{L}$ Tris-HCl ( $\mathrm{pH} 8.3$ ), $50 \mathrm{mmol} / \mathrm{L} \mathrm{KCl}, 1.5 \mathrm{mmol} / \mathrm{L}$ $\mathrm{MgCl}_{2}$, Taq 酶 $1 \mathrm{U}$,四种 $\mathrm{dNTP}$ 各 $150 \mu \mathrm{mol} / \mathrm{L}$,两个 引物各 $10 \mathrm{pmol} / \mathrm{L}$,DNA 模板约 $100 \mathrm{ng}$ 。反应在 PE2400 型 PCR 仪上进行。循环参数为 $95^{\circ} \mathrm{C}$ 预变性 $4 \mathrm{~min}$, 然后 $95^{\circ} \mathrm{C}$ 变性 $40 \mathrm{~s}, 55^{\circ} \mathrm{C} \sim 58^{\circ} \mathrm{C}$ 复性 $40 \mathrm{~s}, 72$ ${ }^{\circ} \mathrm{C}$ 延伸 $60 \mathrm{~s}$, 共 32 个循环, 最后 $72^{\circ} \mathrm{C} 10 \mathrm{~min}$ 延伸补 齐。扩增产物用 $\mathrm{Wizard}^{\mathrm{TM}}$ PCR Preps DNA 纯化试剂 盒(Promega 公司)纯化,然后在 ABI 310 基因分析 仪上用双脱氧法测序。DNA 序列在 GenBank 中的 注册号是 AY190634-AY190658 (表 1)。

\section{3 序列分析}

用 Clustal W 程序 (Thompson et al. ，1994) 对 DNA 序列进行对位排列, 用 MEGA (Kumar \& Nei , 1993 )中的 Kimura (1980) 双参数模型计算序列变异 和遗传距离, 采用邻接法 ( neighbor-joining, NJ) ( Saitou \& Nei , 1987) 推演系统发生关系。在构建系统 发生树时，应用自举检验(Felsenstein，1985) 估计系 统树中结点的置信值, 同时用单倍型相似性指数建 立种群系统发生树, 单倍型相似性指数 $=2 H /\left(H_{p}+\right.$ $\left.H_{q}\right)$ 其中 $H_{p}$ 和 $H_{q}$ 分别表示种群 $p$ 和 $q$ 的单倍型 数, $H$ 表示种群 $p$ 和 $q$ 的共享单倍型数。单倍型多 样性指数 $H=\left(1-\sum x_{i}^{2}\right) n /(n-1)$;核苷酸多样性 $P$ $=\sum X_{i} X_{j} P_{i j}$, 其中 $X_{i} 、 X_{j}$ 分别表示第 $i$ 和 $j$ 种单倍型 在种群内的频率, $P_{i j}$ 表示第 $i$ 和 $j$ 种单倍型的基因差 异率 (Nei ,1987)。用 SPSS 10.0 软件进行统计分 析。 


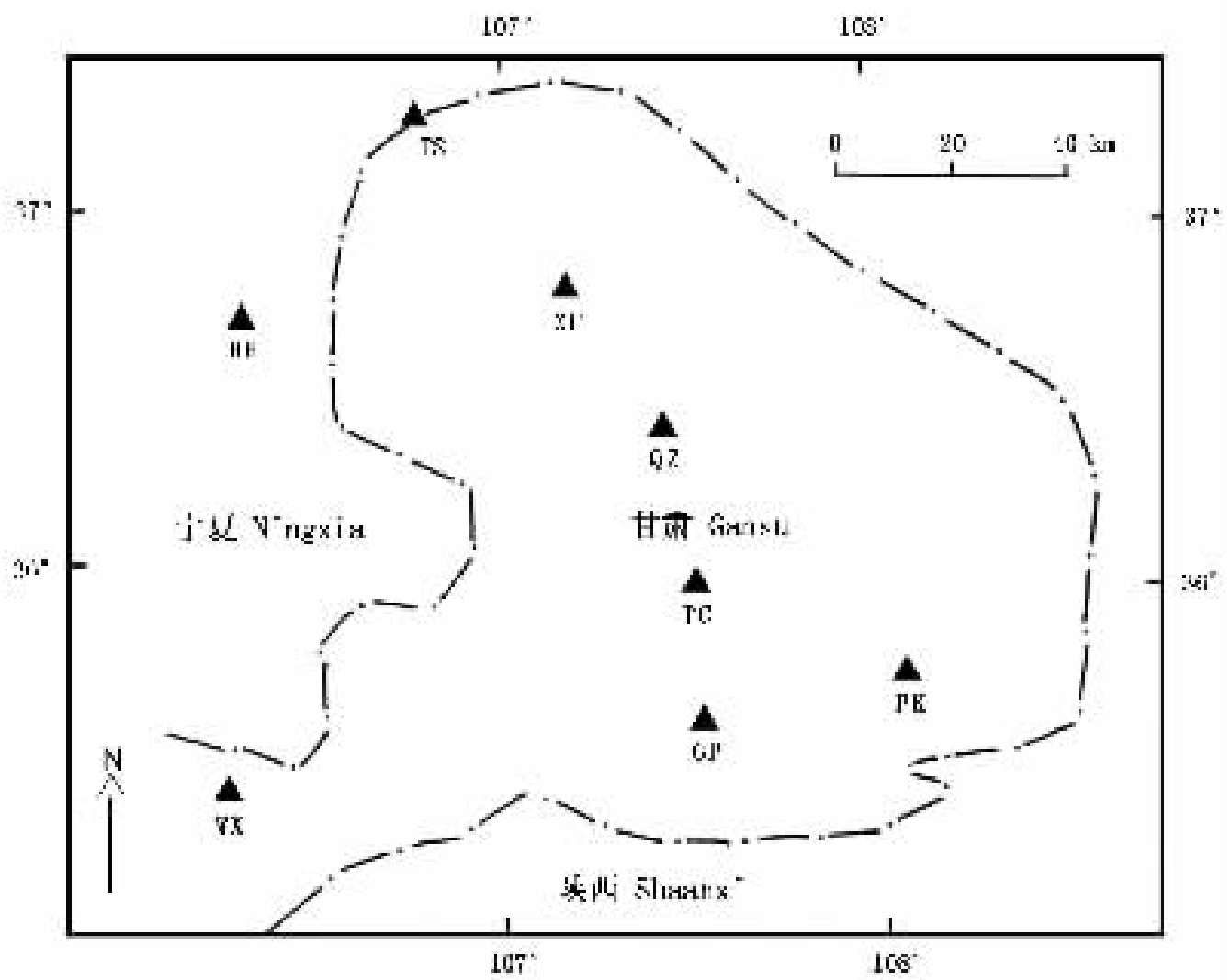

图 1 石鸡 78 个个体的取样地点

种群代号 TS : 甜水 ; XC : 环县西川 ; $\mathrm{QZ}$ : 环县曲子 ; TC : 庆阳铜川 ; PK : 宁县盘克 : GP : 泾川高平 ; WX : 华亭王峡 ; HH : 宁 夏海原红回

Fig. 1 Map showing the sample localities of the 78 individuals of Alectoris chukar.

TS , Tianshui population ; XC , Xichuan population ; QZ , Quzi population ; TC, Tongchuan population ; PK , Panke population ; GP , Gaoping population ; WX , Wangxia population.

\section{2 结果}

\section{1 序列变异}

本文测定 78 个石鸡个体的 $m t D N A$ 控制区高变 I 区和部分 II 区共 $491 \mathrm{bp}$ 的序列, 共发现 24 个变异 位点(占所测序列的 $4.89 \%$ ) 25 种单倍型 (占所测 样本的 $32.05 \%$ ) (表 1)。8 个种群中, 铜川种群的 序列变异率最高, 达 0.47 红回种群的最低, 仅 0.10 (表 2)。

\section{2 种群遗传多样性}

8 个种群中, 铜川种群的单倍型多样性和核甘 酸多样性都最高, 分别达 0.82 和 0.0029 ;红回种群 的最低，仅 0.28 和 0.0008 (表 2)。平均单倍型多样 性 0.67 , 平均核苷酸多样性 0.0017 。

\section{3 种群系统发生}

陇东高原 8 个石鸡种群共享 1 种单倍型 C1 ( 表
2 ）,说明它们来自共同祖先。25种单倍型在 NJ 树 (图 2 ) 上相互混杂, 没有按种群形成独立的分枝, 但 聚成两个枝 $\mathrm{A} 1$ 和 $\mathrm{A} 2$, 用单倍型相似性指数构建的 种群系统发生树 (图 3 ) 很明显聚成两个不同集群 $\mathrm{D} 1$ 和 $\mathrm{D} 2$,与 $\mathrm{NJ}$ 树的 $\mathrm{A} 1$ 和 $\mathrm{A} 2$ 基本对应一致。两个 集群的单倍型相似性仅 0.15 , 遗传差异则达 $0.43 \%$,单因素方差检验显示两个集群间遗传变异 差异显著 $\left(F=5.02>F_{0.05}(14,1)=4.06\right)$ 。两个集 群的平均单倍型多样性分别是 0.62 和 0.72 , 平均 核苷酸多样性分别是 0.0018 和 0.0016 。

\section{3 讨论}

陇东高原石鸡种群的平均单倍型多样性是 0.67 , 平均核苷酸多样性是 0.0017 ,比其近缘种欧 石鸡 (A. graeca) (Lucchini \& Randi, 1998)的相应值 0.49 和 0.0014 都高。但 8 个种群中, 红回种群的 
表 1 石鸡的线粒体 DNA 单倍型、变异位点及其 GenBank 序列号

Table 1 mtDNA haplotypes, variable sites and GenBank accession numbers in Alectoris chukar

\begin{tabular}{|c|c|c|c|}
\hline $\begin{array}{l}\text { 单倍型 } \\
\text { Haplotype }\end{array}$ & $\begin{array}{c}\text { 样品编号 } \\
\text { Sample code }\end{array}$ & $\begin{array}{c}\text { 变异位点 Variable sites in sequence } \\
000000012222223344444444 \\
000114960012460257888899 \\
236371141719990210238901 \\
\end{array}$ & $\begin{array}{c}\text { GenBank 序列号 } \\
\text { GenBank accession number }\end{array}$ \\
\hline $\mathrm{C} 1$ & $*$ & ACCACCTCCCTACGCCCCTCACAT & AY190634 \\
\hline $\mathrm{C} 2$ & $* *$ & $\ldots \ldots$.............. & AY190635 \\
\hline $\mathrm{C} 3$ & TS1 & 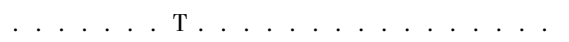 & AY190636 \\
\hline $\mathrm{C} 4$ & $\operatorname{TS} 3,5$ & 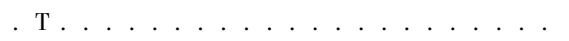 & AY190637 \\
\hline C5 & TS10,QZ5 ,8 & $\ldots \ldots$. . . . . . & AY190638 \\
\hline C6 & TS8, ,GP13 & 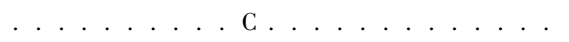 & AY190639 \\
\hline $\mathrm{C} 7$ & XC6 & $\ldots \ldots$. . . . . . A & AY190640 \\
\hline $\mathrm{C} 8$ & XC7 & $\ldots \ldots \mathrm{G} . \mathrm{A}$ & AY190641 \\
\hline C9 & $\mathrm{XC9}$ & $\ldots \ldots$. . . . . . . . . & AY190642 \\
\hline C10 & QZ1 & $\ldots \ldots$. . . . . . . & AY190643 \\
\hline C11 & QZ7 & 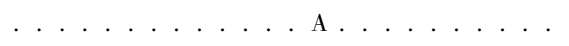 & AY190644 \\
\hline C12 & TC2 ,PK5 & $\ldots \ldots \ldots$ & AY190645 \\
\hline C13 & TC4 5 & $\ldots \ldots$. . . . . . . . & AY190646 \\
\hline C14 & TC8 & $\ldots \ldots \mathrm{TA} \ldots \mathrm{T} \ldots \ldots \mathrm{AT}$. & AY190647 \\
\hline C15 & TC9 & $\ldots \ldots \mathrm{G} \ldots \mathrm{T} \ldots \mathrm{T} \cdot \mathrm{ATA}$ & AY190648 \\
\hline C16 & $\mathrm{TC} 10, \mathrm{WX} 4$ & $\ldots \ldots \ldots \ldots \mathrm{A} \mathrm{T} \mathrm{A}$ & AY190649 \\
\hline $\mathrm{C} 17$ & PK1 $2,3,7,9$ & $\ldots \ldots \ldots \ldots \ldots \ldots$ & AY190650 \\
\hline C18 & WX3 & $\ldots \ldots \mathrm{G} \ldots \ldots \ldots \ldots$ & AY190651 \\
\hline C19 & WX6 & . . . С. . . А. . . . . & AY190652 \\
\hline $\mathrm{C} 20$ & WX8 & . . . . . . A & AY190653 \\
\hline $\mathrm{C} 21$ & GP5 & $\mathrm{C} \ldots \ldots . \ldots$ & AY190654 \\
\hline $\mathrm{C} 22$ & GP6 & $\ldots \mathrm{T} \ldots \ldots \mathrm{T} \ldots \ldots$ & AY190655 \\
\hline $\mathrm{C} 23$ & GP8 & $\ldots \mathrm{TC} \ldots \ldots . \ldots$ & AY190656 \\
\hline $\mathrm{C} 24$ & GP14 & $\ldots \ldots \mathrm{G} \ldots \ldots \mathrm{G} \ldots \ldots$ & AY190657 \\
\hline $\mathrm{C} 25$ & HH6 & $\ldots \mathrm{A} \ldots \mathrm{T} \ldots \mathrm{A}$ & AY190658 \\
\hline
\end{tabular}

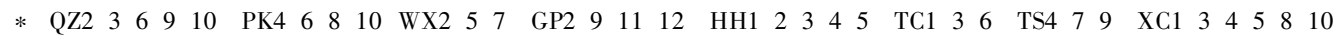

* * :GP1 $3,4,7,10 ; \mathrm{TC} 7$; TS2 6 ; XC2 ; QZ4 ; WX1

单倍型多样性和核甘酸多样性都最低,仅 0.28 和 0.0008 ,其原因可能在于: (1) 红回地处石鸡和大石 鸡分布的接触地带,是边缘种群，受奠基者效应 (founder effect) 影响，其遗传多样性低; (2) 红回种 群与邻近种群仅共享一种单倍型 C1 ( 表 2) ,由于遗 传隔离，受近交衰退的影响严重; (3) 自然选择的作 用，红回种群处于石鸡分布的边缘，受自然选择的压 力比较大, 不适应环境的基因型被淘汰。

宿兵等 (1996) 对我国黑冠长臂猿 (Hylobates concolor) 11 个个体线粒体 DNA 的 $159 \mathrm{bp}$ 进行分 析，确定 3 个种和 2 个亚种，据此提出中国黑冠长臂 猿有 5 个 ESUs。Houlden et al. (1999) 分析澳大利 亚树袋熊 (Phascolarctos cinereus) 地理种群线粒体基 因序列时发现，它是由多个管理单元构成的单一的 进化显著单元。Barratt et al. (1999) 对英国红松鼠 (Sciurus vulgaris) 的研究也得出类似的结果。Kark et al. (1999) 运用等位酶技术确定以色列的石鸡优 先保护种群时, 发现生态交错带的种群具有很高的 
表 28 个石鸡种群的样本量、单倍型类型、单倍型频率、序列变异率、单倍型多样性和核苷酸多样性

Table 2 The sample size, haplotype, haplotype frequency, sequence variation, haplotype diversity and nucleotide diversity of eight $A$. chukar populations

\begin{tabular}{|c|c|c|c|c|c|c|}
\hline $\begin{array}{c}\text { 种 群 } \\
\text { Population }\end{array}$ & $\begin{array}{l}\text { 样本量 } \\
\text { Sample } \\
\text { size }\end{array}$ & $\begin{array}{c}\text { 单倍型类型 } \\
\text { Haplotype }\end{array}$ & $\begin{array}{c}\text { 单倍型频率 } \\
\text { Haplotype } \\
\text { frequency }\end{array}$ & $\begin{array}{c}\text { 序列变异率 ( \%) } \\
\text { Sequence } \\
\text { variation }\end{array}$ & $\begin{array}{c}\text { 单倍型多样性 } \\
\text { Haplotype } \\
\text { diversity }\end{array}$ & $\begin{array}{c}\text { 核苷酸多样性 } \\
\text { Nucleotide } \\
\text { diversity }\end{array}$ \\
\hline 甜水种群 & 10 & C1 & 0.30 & 0.18 & 0.80 & 0.0015 \\
\hline Tianshui Population & & $\mathrm{C} 2$ & 0.20 & & & \\
\hline \multirow[t]{4}{*}{ ( TS ) } & & $\mathrm{C} 3$ & 0.10 & & & \\
\hline & & $\mathrm{C} 4$ & 0.20 & & & \\
\hline & & C5 & 0.10 & & & \\
\hline & & C6 & 0.10 & & & \\
\hline 西川种群 & 10 & $\mathrm{C} 1$ & 0.60 & 0.14 & 0.60 & 0.0012 \\
\hline Xichuan Population & & $\mathrm{C} 2$ & 0.10 & & & \\
\hline \multirow[t]{3}{*}{$(\mathrm{XC})$} & & $\mathrm{C} 7$ & 0.10 & & & \\
\hline & & $\mathrm{C} 8$ & 0.10 & & & \\
\hline & & C9 & 0.10 & & & \\
\hline 曲子种群 & 10 & $\mathrm{C} 1$ & 0.50 & 0.12 & 0.68 & 0.0010 \\
\hline Quzi Population & & $\mathrm{C} 2$ & 0.10 & & & \\
\hline \multirow[t]{3}{*}{$(\mathrm{QZ})$} & & C5 & 0.20 & & & \\
\hline & & $\mathrm{C} 10$ & 0.10 & & & \\
\hline & & $\mathrm{C} 11$ & 0.10 & & & \\
\hline 铜川种群 & 10 & $\mathrm{C} 1$ & 0.30 & 0.47 & 0.82 & 0.0029 \\
\hline Tongchuan Population & & $\mathrm{C} 2$ & 0.10 & & & \\
\hline \multirow[t]{5}{*}{$(\mathrm{TC})$} & & $\mathrm{C} 12$ & 0.10 & & & \\
\hline & & C13 & 0.20 & & & \\
\hline & & C14 & 0.10 & & & \\
\hline & & C15 & 0.10 & & & \\
\hline & & $\mathrm{C} 16$ & 0.10 & & & \\
\hline 盘克种群 & 10 & $\mathrm{C} 1$ & 0.40 & 0.22 & 0.58 & 0.0012 \\
\hline Panke Population & & $\mathrm{C} 12$ & 0.10 & & & \\
\hline$(\mathrm{PK})$ & & $\mathrm{C} 17$ & 0.50 & & & \\
\hline 高平种群 & 14 & $\mathrm{C} 1$ & 0.28 & 0.29 & 0.78 & 0.0022 \\
\hline Gaoping & & $\mathrm{C} 2$ & 0.36 & & & \\
\hline Population & & C6 & 0.07 & & & \\
\hline \multirow[t]{4}{*}{ ( GP) } & & $\mathrm{C} 21$ & 0.07 & & & \\
\hline & & $\mathrm{C} 22$ & 0.07 & & & \\
\hline & & $\mathrm{C} 23$ & 0.07 & & & \\
\hline & & $\mathrm{C} 24$ & 0.07 & & & \\
\hline 王峡种群 & 8 & $\mathrm{C} 1$ & 0.38 & 0.25 & 0.78 & 0.0021 \\
\hline Wangxia Population & & $\mathrm{C} 2$ & 0.12 & & & \\
\hline \multirow[t]{4}{*}{$(\mathrm{WX})$} & & C16 & 0.12 & & & \\
\hline & & C18 & 0.12 & & & \\
\hline & & C19 & 0.12 & & & \\
\hline & & $\mathrm{C} 20$ & 0.12 & & & \\
\hline 红回种群 & 6 & $\mathrm{C} 1$ & 0.83 & 0.10 & 0.28 & 0.0008 \\
\hline $\begin{array}{l}\text { Honghui Population } \\
\text { ( } \mathrm{HH})\end{array}$ & & $\mathrm{C} 25$ & 0.17 & & & \\
\hline
\end{tabular}




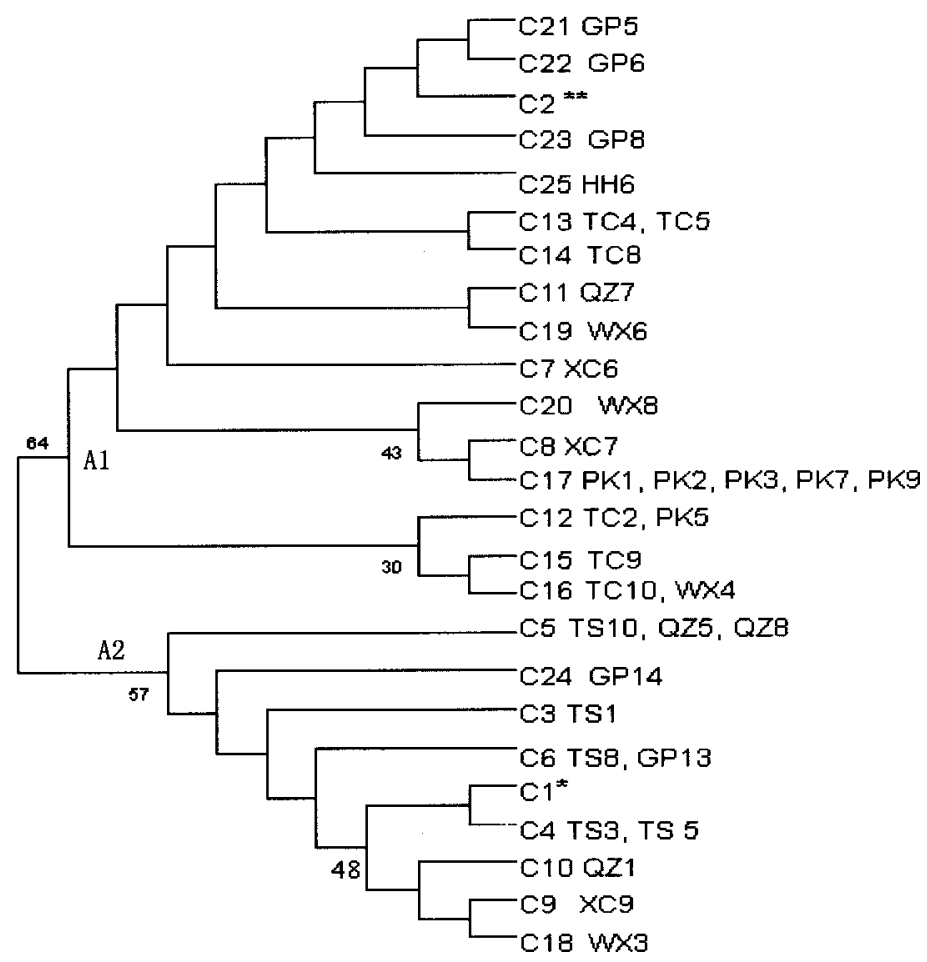

图 2 石鸡 mtDNA D-loop 基因序 列的 NJ (neighbor-joining ) 树 (Kimura-2-parameter)，枝上数字 是经过 1000 次重复抽样的 bootstrap 值 $(\geqslant \mathbf{5 0 \%})$; 各分枝后是单倍 型序号和样本代码, 各单倍型变异 位点见表 1 。

Fig. 2 Neighbor-joining tree constructed from the sequences of mtDNA D-loop in Alectoris chukar based on Kimura-2-parameter distances. Numbers at internodes represent bootstrap values $(\geqslant 50 \%)$ from 1000 replications. The variable sites of each haplotype and sample codes were shown in Table 1 .

* :QZ2 3, 6, 9, 10; PK4, 6, 10 ; WX2 5,7 ; GP2, 9, 11, 12; HH1 2, $3,4,5 ; \mathrm{TC1}, 3,6 ; \mathrm{TS} 4,7,9 ; \mathrm{XC1}, 3$, $4,5,8,10$.

* * :GP1 3 : 4, 10 ; TC7 ; TS2 6 ; $\mathrm{XC2} ; \mathrm{QZ4} ; \mathrm{WX} 1$.

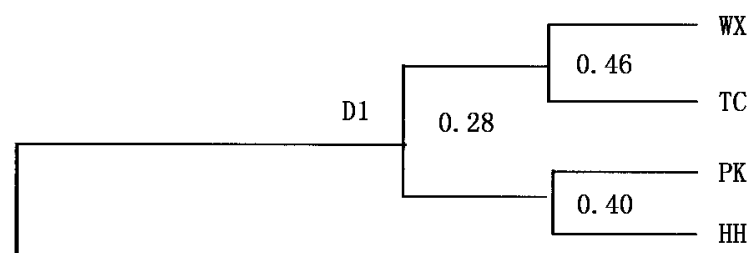

0.15

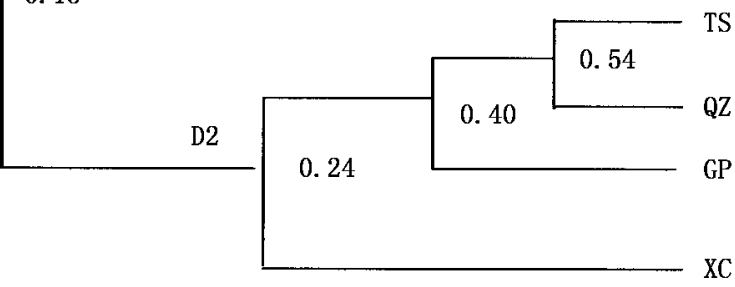

图 3 基于单倍型相似性构建的石鸡种群系统发生树 (树上 数字代表各集群的单倍型相似性, 种群代号同图 1)

Fig. 3 Population phylogeny based on haplotype similarity of Alectoris chukar. Numbers at internodes represent the haplotype similarity between groups. Population codes as in the legend to Fig. 1 .

遗传多样性, 是其他种群遗传变异的来源, 应优先保 护。本文通过分析陇东黄土高原 78 个石鸡样本的 $491 \mathrm{bp}$,发现 8 个种群共享 1 种单倍型 C1 说明它们 来自共同的祖先, 是 1 个单系群,所以它们属于 1 个 $\mathrm{ESU}$ 。同时 8 个种群聚成两个集群, 分别有 13 种和
10 种单倍型为两者特有, 而且其遗传变异差异显著 $\left(F=5.02>F_{0.05}(14,1)=4.06\right)$ 。基于遗传差异， 应把这个高原上的石鸡作为两个 MUs 来进行保护。 8 个种群中, 铜川种群的序列变异率、单倍型多样性 和核苷酸多样性都最高, 应重点保护。

致谢：感谢 Ettore Randi 博士提供引物。

\section{参考文献}

Avise J. C. 1994. Molecular Markers, Natural History and Evolution. Chapman and Hall, New York.

Barratt E. M., Gurnell J., Malarky G., Deaville R. and Bruford M. W. 1999. Genetic structure of red squirrel (Sciurus vulgaris) in the U. K. Molecular Ecology, 8(12): 555 563.

Brown W. M., George M. Jr. and Wilson W. C. 1979. Rapid evolution of animal mitochondrial DNA. Proceedings of the National Academy of Sciences, USA, 76(4) : 1967 - 1971.

Felsenstein J. 1985. Confidence limits on phylogenies: an approach using the bootstrap. Evolution, 39:783-791.

Gyllensten U., Wharton D. and Wilson A. C. 1985. Maternal inheritance of mitochondrial DNA during backcrossing of two species of mice. The Journal of Heredity, 76: 321 - 
324.

Haig S. M. 1998. Molecular contributions to conservation. Ecology, 79(2): 413 - 425 .

Houlden B. A., Costello B. H., Sharkey D., Flower E. V., Melzer A., Ellis W., Carrick F., Baverstock P. R. and Elphinstone M. S. 1999. Phylogeographic differentiation in the mitochondrial control region in the koala, Phascolarctos cinereus. Molecular Ecology, 8: 999 - 1011.

Humphries C. J., Williams P. H. and Vane-Wright R. I. 1995. Measuring biodiversity value for conservation. Annual Review of Ecology and Systematics, 26:93 - 111.

Kark S., Alkon P. U., Safril U. N. and Randi E. 1999. Conservation priorities for chukar partridge in Israel based on genetic diversity across an ecological gradient. Conservation Biology, 13(3) : 542 - 552.

Kimura M. 1980. A simple method for estimating evolutionary rate of base substitutions through comparative studies of nucleotide sequences. Journal of Molecular Evolution, 16: $111-120$.

Kumar S. K. and Nei M. 1993. MEGA, Molecular Evolutionary Genetics Analysis, Version 1.01. University Park, Pennsylvania State University.

Lucchini V. and Randi E. 1998. Mitochondrial DNA sequence variation and phylogeographical structure of rock partridge (Alectoris graeca) populations. Heredity, 31:528 - 536.

Moritz C. 1994. Defining evolutionarily significant units for conservation. Trends in Ecology and Evolution, 9:373 - 375.

Moritz C. 1995. Use of molecular phylogenies for conservation. Philosophical Transactions: Biological Sciences B, 349: 113 -118 .

Nei M. 1987. Molecular Evolutionary Genetics. Columbia University Press, New York.

O'Brien S. J. and Mayr E. 1991. Bureaucratic mischief: recognizing endangered species and subspecies. Science, 251: $1187-1188$.

Randi E. and Alkon P. U. 1994. Genetic structure of chukar (Aletoris chukar) population in Israel. The Auk, 111(2): $216-226$.

Randi E. and Lucchini V. 1998. Organization and evolution of the mitochondrial DNA control region in the avian genus Alectoris. Journal of Molecular Evolution, 47 (4): 449 462.

Ryder O. A. 1986. Species conservation and systematics: the dilemma of subspecies. Trends in Ecology and Evolution, 1: $9-10$.

Saitou N. and Nei M. 1987. The neighbor-joining method: a new method for reconstruction phylogenetic trees. Molecular Biology and Evolution, 4: 406 - 425.

Shi L-M(施立明), Jia X(贾旭) and Hu Z-A(胡志昂). 1993. Genetic diversity. In: Chen L-Z(陈灵芝) ( ed.), Current Status and Conservation of Biodiversity in China (中国的生 物多样性一一现状及其保护对策). Science Press, Beijing, $31-113$. ( in Chinese)

Su B. (宿兵), Kressirer P., Monda K., Wang W. (王文), Jiang X-L(蒋学龙), Wang Y-X (王应祥), Woodruff D. S. and Zhang Y-P(张亚平). 1996. Mitochondrial DNA variation and molecular phylogeny of Chinese concolor gibbons with non-invasive DNA genotyping. Science in China (Series C) (中国科学 C 辑), 26: $414-419$. (in Chinese)

Thompson J. D., Higgins D. G. and Bibson T. J. 1994. CLUSTAL W: improving the sensitivity of progressive multiple sequence alignment through sequence weighting position-specific gap penalties and weight matrix choice. Nucleic Acid Research, 22: 4673 - 4680.

Wang X-T(王香亭)。1991. Vertebrate Fauna of Gansu( 甘肃脊 椎动物志). Gansu Science and Technology Press, Lanzhou, 87 - 395. (in Chinese)

Wang W(王文). 1998. Molecular phylogenetics and biodiversity conservation. Chinese Biodiversity (生物多样性), 6 (2) : 138 - 142. (in Chinese)

W ang J-B(王静波), Hu C-L(胡长龙) and Xu H-F( 徐宏发). 2001. Applications of mitochondrial DNA variability analysis in zoological conservation biology. Biodiversity Science (生物多样性), 9(2): 181-187. (in Chinese)

Yang Y-H(杨玉慧) and Li Y-M(李义明). 2001. Molecular ecology and animal biodiversity conservation. Biodiversity Science(生物多样性), 9(3)：284-293. (in Chinese) 